\title{
Insulin Resistance in Chronic Kidney Disease: Is There a Distinct Role of Vitamin D?
}

\author{
Heba A. Attea ${ }^{{ }^{*}}$, Alaa El Din S. Abd El Hamid², Mohammed M. Keshawy ${ }^{1}$, \\ Mohamed S. Khedr ${ }^{1}$ \\ 'Department of Internal Medicine, faculty of Medicine, Suez Canal University; ${ }^{2}$ Department of Clinical Pathol- \\ ogy, faculty of Medicine, Suez Canal University
}

\begin{abstract}
Background: Chronic kidney disease (CKD) is associated with significant morbidity and mortality. Many factors including inflammation, oxidative stress, and vitamin D deficiency contribute to insulin resistance (IR). Vitamin D requires attention because of the kidney's involvement in vitamin D metabolism, the high prevalence of vitamin D deficiency in CKD, and the effect of vitamin D that may cover multiple factors contributing to IR in CKD, as inflammation. Aim: To Identify the role of vitamin $D$ deficiency in diabetic kidney disease (DKD) in type 2 diabetes mellitus (T2DM) patients. Patients and Methods: The study was performed as a cross-sectional study including 84 patients with T2DM admitted in Suez Canal University Hospitals. Results: In diabetics with normal kidney function $64.3 \%$ of patients had sufficient level of vitamin D, while $14.3 \%$ of patients had vitamin $D$ deficiency. In diabetics with late nephropathy $46.4 \%$ of patients had vitamin $D$ deficiency with significant relationship between the progress of DN and vitamin $D$ level ( $p$ value $\leq$ 0.05). Conclusion: vitamin D deficiency is strongly associated with DN.
\end{abstract}

Keywords: chronic kidney disease, insulin resistance, vitamin D.

\section{Introduction}

Type 2 diabetes mellitus (T2DM) has become a global health care problem. In 2019, there were 463 million diabetes patients worldwide, with 4.2 million diabetes related deaths. Efforts have been devoted to finding innovative approaches for diabetes prevention and treatment ${ }^{(1)}$. The strong and consistent inverse associations between blood 25(OH)D concentration and incident diabetes reported in observational studies are supported by data on biological plausibility from mechanistic studies and raise the possibility that optimizing vitamin $D$ status may reduce the risk of $\mathrm{T}_{2} \mathrm{DM}^{(2)}$. Observational studies have indicated an association between vitamin $D$ deficiency and the onset and progression of DM as well as future macro-vascular events. Moreover, in vivo, and in vitro studies have proposed potential roles of vitamin D in glucose metabolism, e.g., stimulating insulin secretion via the vitamin $D$ receptor on pancreatic beta cells; modulating immune responses and lowering systematic inflammation; and reducing peripheral insulin resistance through vitamin $D$ receptors in the muscles and liver $(3,4)$. It is documented that vitamin $D$ acts as a neg- 
tive endocrine regulator for the renin-angiotensin system and this would indicate a potential involvement of vitamin $D$ in the progressive loss of renal function. Another possible mechanism that could relate vitamin $D$ and progression of renal disease is the involvement of vitamin $D$ in cell cycle regulation and cell differentiation ${ }^{(5)}$.

\section{Patients and Methods}

The study was performed as a cross- sectional study. This study included 84 patients with T2DM admitted in Nephrology care services at Suez Canal University Hospitals. They were divided as 3 groups: control group (diabetics with normal kidney function), the case groups are of diabetics with early nephropathy (i.e., GFR $\geq 60$ $\mathrm{ml} / \mathrm{min}$ ), and diabetics with late nephropathy (i.e., GFR < $60 \mathrm{ml} / \mathrm{min}$ ). CKD is defined according to the K/DOQI definitions ${ }^{(6)}$. Serum creatinine was determined by routine techniques using an automated analyzer (COBAS 6000 Automated Chemistry Analyzer), used in SCU clinical pathology laboratory) and e GFR was calculated using the Chronic Kidney Disease Epidemiology collaboration equation $(7)$. Consistent with American Diabetes Association guidelines, albumin and creatinine was measured in a random spot urine sample. These measurements were used to calculate the urinary albumin-to-creatinine ratio. Nephropathy was defined as a urinary albumin-to-creatinine ratio $\geq 30 \mathrm{mg} / \mathrm{g}^{(8)}$. Lipid profile including serum total cholesterol, triglyceride, low density lipoprotein (LDL) and highdensity lipoprotein (HDL). Study participants are considered to have dyslipidemia when cholesterol level is $\geq 200 \mathrm{mg} / \mathrm{dl}$ (LDL $\geq 129 \mathrm{mg} / \mathrm{dl}$, HDL <40mg/dl for men and $<50 \mathrm{mg} / \mathrm{dl}$ for women) or triglyceride level is $\geq 150 \mathrm{mg} / \mathrm{dl}^{(9)}$. Insulin resistance was assessed by the homoeostasis model (HOMA). The insulin level was measured by mean of competitive enzyme immunoassay with a double antibody procedure using Insulin ELISA Kit (BDIN31-BA), 96 Tests, Germany. Patient with IR defined by HOMA-IR $>2^{(10)}$. Vitamin D level assay was done by $25(\mathrm{OH})$ Vitamin D ELISA (BD200BA), 96 tests, (Homburg, Germany). Patients with vitamin D level $\geq 30 \mathrm{ng} / \mathrm{ml}, 21$ to $29 \mathrm{ng} / \mathrm{ml}, \leq 20 \mathrm{ng} / \mathrm{ml}$ are considered to have normal vitamin D level, insufficiency or deficiency respectively ${ }^{(11)}$. Both sexes and adult patients were included in the study. Exclusion criteria include hypertension, chronic liver disease, T1DM and usage of drugs as insulin or insulin sensitizers or vitamin D analogues. The collected data was coded, and the statistical analysis done using the SPSS, version 24. The Ethics Committee of Suez Canal University approved the study on 23/10/2017 (project number: 3252).

\section{Results}

The results showed that in diabetics with normal kidney function $64.3 \%$ of patients had sufficient level of vitamin $D$, while $14.3 \%$ of patients had vitamin $D$ deficiency. In diabetics with late nephropathy $46.4 \%$ of patients had vitamin $D$ deficiency with significant relationship between the progress of diabetic nephropathy and vitamin D deficiency as shown in table (1). In diabetics with normal kidney function $71.4 \%$ of patients were IS ( $90 \%$ of those had sufficient level of vitamin D), while $28.6 \%$ of patients were IR and none of them had sufficient vitamin $D$ level. While In diabetics with early nephropathy $60.7 \%$ of patients were IS (76.5\% of those had sufficient level of vitamin D), while $39.3 \%$ of patients were IR (18.2\% of those had sufficient vitamin D level). In diabetics with late nephropathy $21.4 \%$ of patients were IS $(83.3 \%$ of those had sufficient level of vitamin $D$ ), while $78.6 \%$ of patients were IR (only $4.5 \%$ of 
those had sufficient vitamin $\mathrm{D}$ level). This showed strong statistically significant relationship between vitamin $D$ deficiency and insulin resistance $(p<0.001)$ as shown in table (2). The results showed that vitamin $d$ deficiency is positively associated with occurrence of insulin resistance, such that adjusting for the other variables in the model, for each increase in level of vitamin d deficiency, occurrence of insulin resistance is predicted to increase by 4.4 units.

Table 1: Comparison between the three studied groups according to Vitamin D level

\begin{tabular}{|c|c|c|c|c|c|c|c|c|}
\hline \multirow[t]{2}{*}{ Vit. D level } & \multicolumn{2}{|c|}{$\begin{array}{l}\text { Diabetics with } \\
\text { early DN } \\
(n=28)\end{array}$} & \multicolumn{2}{|c|}{$\begin{array}{l}\text { Diabetics with } \\
\text { late DN } \\
(n=28)\end{array}$} & \multicolumn{2}{|c|}{$\begin{array}{l}\text { Diabetics with normal } \\
\text { kidney function } \\
(n=28)\end{array}$} & \multirow[t]{2}{*}{$\begin{array}{l}\text { Test of } \\
\text { Sig. }\end{array}$} & \multirow[t]{2}{*}{$\mathrm{p}$} \\
\hline & No. & $\%$ & No. & $\%$ & No. & $\%$ & & \\
\hline $\begin{array}{l}\text { Sufficient level } \\
(\geq 30 \mathrm{ng} / \mathrm{ml})\end{array}$ & 15 & 53.6 & 6 & 21.4 & 18 & $64 \cdot 3$ & \multirow{3}{*}{$x^{2}=13.2$} & \multirow{3}{*}{0.01} \\
\hline $\begin{array}{l}\text { Insufficiency } \\
(21-29 \mathrm{ng} / \mathrm{ml})\end{array}$ & 8 & 28.6 & 9 & 32.1 & 6 & 21.4 & & \\
\hline $\begin{array}{l}\text { Deficiency } \\
(\leq 20 \mathrm{ng} / \mathrm{ml})\end{array}$ & 5 & 17.9 & 13 & 46.4 & 4 & $14 \cdot 3$ & & \\
\hline Range & \multicolumn{2}{|c|}{$4.60-45.20$} & \multicolumn{2}{|c|}{$4.60-50.0$} & \multicolumn{2}{|c|}{$4.40-57.80$} & \multirow[b]{2}{*}{$\mathrm{H}=0.951$} & \multirow[b]{2}{*}{0.0377} \\
\hline Mean \pm SD. & \multicolumn{2}{|c|}{$21.94 \pm 17.26$} & \multicolumn{2}{|c|}{$17.30 \pm 15.86$} & \multicolumn{2}{|c|}{$28.09 \pm 11.70$} & & \\
\hline
\end{tabular}

$\chi^{2}$ : Chi square test; $\mathrm{H}$ : $\mathrm{H}$ for Kruskal Wallis test; $p: p$ value for comparing between the studied groups;

*: Statistically significant at $p \leq 0.05$

This association is highly statistically significant $(p<0.001)$. Other variables are not statistically significantly associated with occurrence of insulin resistance as shown in table (3). Also, the study results showed that most of the study participants with vitamin D level $<30 \mathrm{ng} / \mathrm{ml}$ are IR, while most of those with vitamin D level $30 \mathrm{ng} / \mathrm{ml}$ or more are IS as shown in figure (1).

\section{Discussion}

The current diabetes pandemic has emerged as a global health burden. Despite accumulating evidence supporting the prevention of obesity and related metabolic disorders, the number of diabetic patients is rapidly increasing, particularly in middle- and low-income countries ${ }^{(12)}$. It is a major concern that diabetes is associated with the development of micro and macro- vascular complications. Diabetic kidney disease (DKD) is the leading cause of endstage renal disease and is therefore a critical issue for healthcare systems ${ }^{(8)}$. In this study, the results show significant relationship between the progress of diabetic nephropathy and vitamin $D$ deficiency $(p=$ 0.01) which agree with the results of a study done in 2019 that showing that serum levels of TNF- $\alpha$ and FBS, and AGEs significantly decreased $(P<0.004)$ in patients receiving vitamin $D(P<0.001)^{(13)}$. The study results are supported by a study done in Egypt in 2019 showing that vitamin D was significantly deficient in diabetic patients in comparison with the control healthy group and it is significantly deficient in DM type 2 with microalbuminuria compared with DM without microalbuminuria ( $p<0.001$ ). These findings indicated that there is a potential role of vitamin $D$ in diabetic 
nephropathy pathogenesis and progress of diabetic nephropthy ${ }^{(14)}$. The present study is in agreement with a Saudi study that reported that there is an overwhelming prevalence of vitamin D deficiency in the diabetic patients and there is associa- tion of low vitamin D status with poor glycemic control and atherogenic lipid profile suggests a role of vitamin $D$ in the control of type 2 DM and dyslipidemia and the importance of early detection of its deficiency and vitamin D supplementa-tion ${ }^{(15)}$.

Table 2: Relation between HOMA IR and Vitamin D level in each group

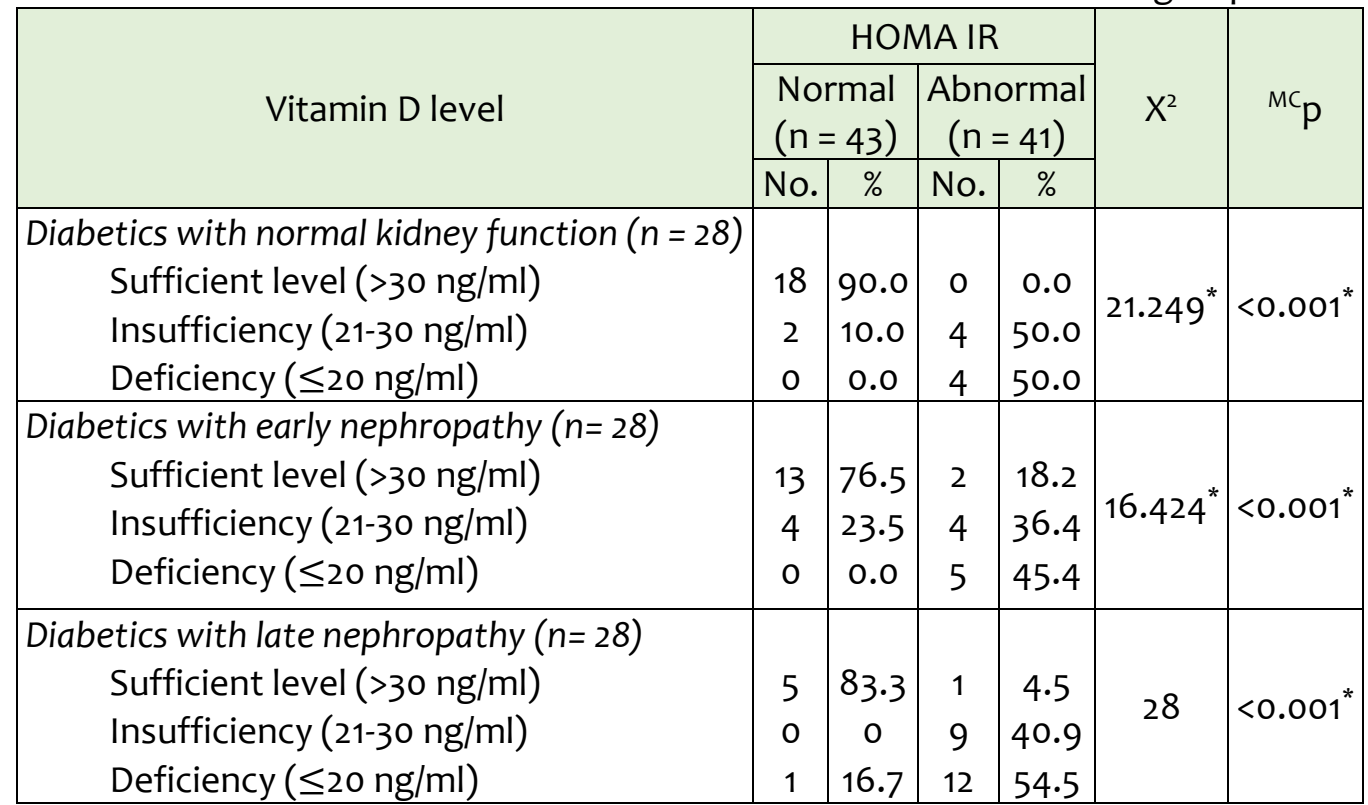

Table 3: Regression analysis of HOMA IR factors among study participants

\begin{tabular}{|ll|c|c|c|}
\hline & & B & Wald & Sig. \\
\hline$\bullet$ & BMI & $-.479-$ & .496 & .481 \\
\hline$\bullet$ & Dyslipidemia & 1.090 & 1.136 & .286 \\
\hline$\bullet$ & Duration of DM & .117 & .016 & .899 \\
\hline$\bullet$ & W/H ratio & .577 & .689 & .406 \\
\hline$\bullet \quad$ Gender & $-2.090-$ & 2.567 & .109 \\
\hline$\bullet \quad$ Vitamin D level & 4.442 & 13.052 & $0.0001^{*}$ \\
\hline$\bullet$ & Age & $-.007-$ & .012 & .914 \\
\hline$\bullet \quad$ Constant & $-1.978-$ & .312 & .576 \\
\hline \multicolumn{4}{|l}{ *Chi square test significant ( $p$ value <0.001), $R^{2}(0.59)}$. \\
\hline
\end{tabular}

Another study concluded that patients with type 2 DM have low 25(OH) vitamin D3 level compared to normal individuals. This study reported the negative association of $25(\mathrm{OH})$ vitamin D3 with glycemic control and its irrelevance to the pro-inflammatory markers suggesting that vitamin D may be an important determinant in the pathogenesis of type 2 DM. Hence, cautious Vitamin D supplementation may have a therapeutic potential in management of Type $2 \mathrm{DM}$ and prevention of diabetic vascular complications ${ }^{(16)}$. Also, another study by Ahmadi and his colleagues showed that the level of 
$25(\mathrm{OH}) \mathrm{D}$ in diabetic patients receiving vitamin $D$ is significantly increased, but there was no significant decrease in proteinuria or a change in GFR after 3 months of treatment. This may be due to presence of dif ferent diabetes-related metabolic factors that increase oxidative stress in diabetic patients as hyperglycemia (uncontrolled blood glucose level), hypertension, dyslipidemia and obesity ${ }^{(17)}$.

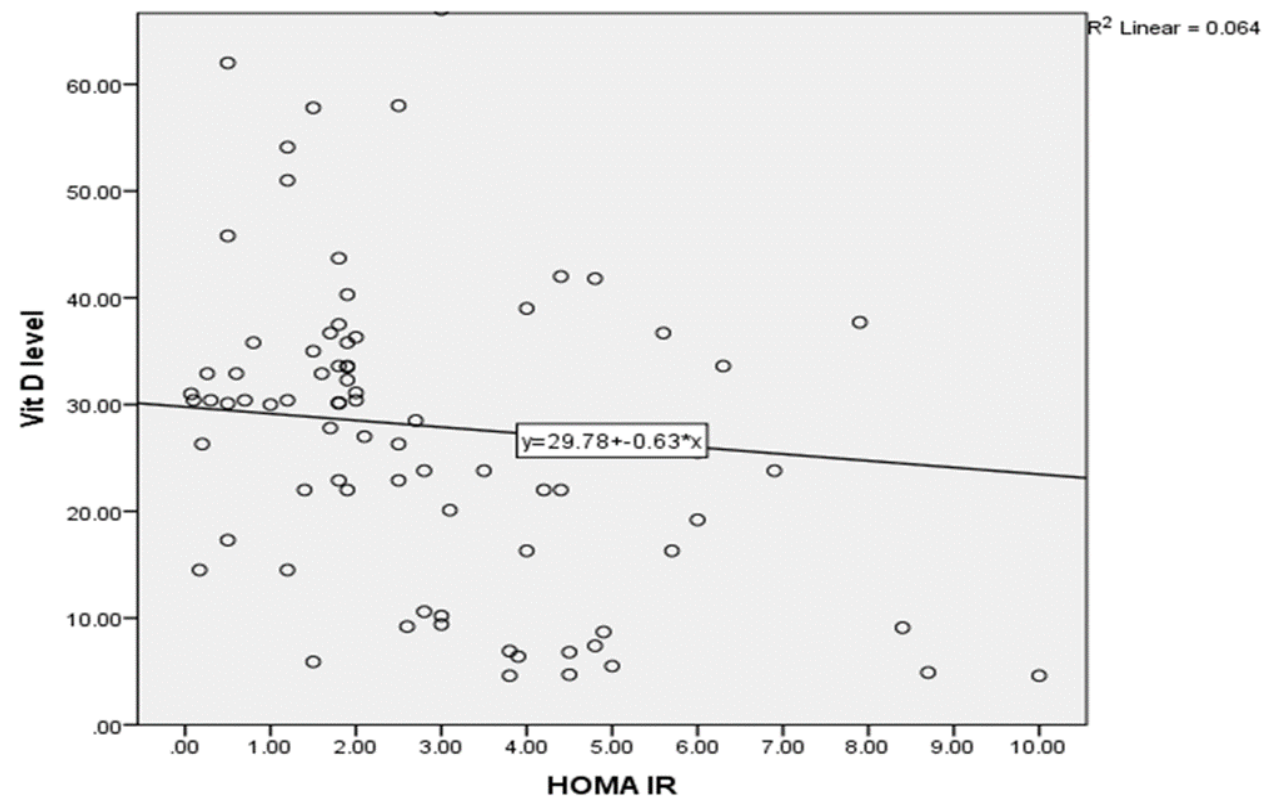

Figure 1: Correlation between HOMA IR and Vit D level among study participants

The present study showed that $53.6 \%$ of diabetics with early nephropathy and $21.4 \%$ of diabetics with late nephropathy had sufficient levels of vitamin D, while $14.3 \%$ of diabetic patients with normal kidney function had vitamin $\mathrm{D}$ deficiency. This can be explained by the presence of a lot of factors that impact the vitamin D status as the synthesis in the skin and absorption of vitamin D, such as sunlight exposure and sea food intake that may have effects on vitamin D status in such categories of patients as well as almost diabetics with eGFR $<60$ $\mathrm{ml} / \mathrm{min} / 1.73 \mathrm{~m}^{2}$ receiving vitamin $D$ supplementation. In the current study, according to HOMA- IR, $28.6 \%, 78.6 \%$ of patients had IR in diabetic patients with normal kidney function, diabetic patients with late nephropathy respectively with significant relationship between the progress of diabetic nephropathy and IR. These results are in accordance with these observations, and it supports the notion that kidney disease is associated with insulin resistance, hyperinsulinemia and hyperglycemia, and that insulin resistance might be is an important factor in the cause and progress of CKD. Kornélia Štefíková and her collegues in 2012 demonstrated that CKD is accompanied by insulin resistance even in stages 2 and 3. This study identified a strong and significant relationship between IR, renal function and proteinuria(18). The current study showed that in diabetics with normal kidney function $90 \%$ of patients who were IS had sufficient level of vitamin $D$, while $50 \%$ of those who were IR had vitamin D deficiency. In diabetics with early nephropa- 
thy $76.5 \%$ of patients who were IS had sufficient level of vitamin D, while $45.5 \%$ of those who were IR had vitamin $D$ deficiency. In diabetics with late nephropathy $83.3 \%$ of patients who were IS had sufficient level of vitamin D, while $54.5 \%$ of those who were IR had vitamin $D$ deficiency. This shows strong statistically significant relationship between vitamin $D$ level deficiency and IR ( $p$ value < 0.001). The present study results agree with a previous study that reported a statistically significant decrease in Fasting serum insulin levels ( $p<0.0031)$ and decrease in IR ( $p$ $<0.0001)$ on maintaining sufficient levels of Vitamin D in the Vitamin D deficient type 2 diabetics in Indian population. Vitamin D supplementation in Diabetics could be beneficial in management of Type $2 \mathrm{DM}^{(19)}$. Finally, there is significant relationship between the progress of diabetic nephropathy and vitamin $D$ deficiency, IR, dyslipidemia and obesity. The current study demonstrated that vitamin $\mathrm{D}$ deficiency is positively associated with insulin resistance, such that adjusting for the other factors as obesity, dyslipidemia and duration of having DM. This association is highly statistically significant ( $p<0.001)$. The current study has some limitations since it is a single-center study with a relatively small number of patients.

\section{Conclusion}

Vitamin D requires particular attention because of the significant relationship between vitamin $D$ deficiency and the progress of diabetic nephropathy. Also, vitamin D control multiple factors that contribute to IR in diabetics, such as inflammation and oxidative stress.

\section{References}

1. Rhys W, Stephen C, Joe C. et al. IDF Atlas $9^{\text {th }}$ Edition 2019.
2. Angellott E, \& Pittas AG. The role of vitamin $D$ in the prevention of type 2 diabetes: to $D$ or not to $D$. Endocrinology, 2017, 158(7), 2013-2021.

3. Park S, Kim DS, Kang S. Vitamin D deficiency impairs glucose-stimulated insulin secretion and increases insulin resistance by reducing PPAR- $\gamma$ expression in nonobese Type 2 diabetic rats. J Nutrit Biochem, 2016, 27, 257265.

4. Li X, Liu Y, Zheng Y. et al. The effect of vitamin $D$ supplementation on glycemic control in type 2 diabetes patients: a systematic review and metaanalysis. Nutrients, 2018, 10(3), 375.

5. Christakos S, Dhawan P, Shen Q. et al. New insights into the mechanisms involved in the pleiotropic actions of 1, 25dihydroxyvitamin D3. Ann NY Acad Sci, 2006, 1068(1), 194-203.

6. National Kidney Foundation Kidney Disease Outcome Quality Initiative (K/DOQI) Advisory Board. K/DOQI clinical practice guidelines for chronic kidney disease. Am J Kidney Dis 2002; 39 [Suppl 2]: S32-S33.

7. Levey AS, Stevens LA, Schmid CH. et al. A new equation to estimate glomerular filtration rate. Ann Intern Med, 2009, 150(9), 604-612.

8. American Diabetes Association. Microvascular Complications and Foot Care: Standards of Medical Care in Diabetes- 2020. Diabetes Care, 43 (Supplement 1), S135-S151.

9. National Institutes of Health. ATP III guidelines at -a-glance quick desk reference. NIH publication, 2001, 013305.

10. Wallace M, Levy C, Matthews R. Use, and abuse of HOMA modeling. Diabetes care, 2004, 27(6), 1487-1495.

11. Holick MF. Vitamin D status: Measurement, interpretation, and clinical application. Ann Epidemiol. 2009; 19(2): 73-78.

12. Atlas D. International Diabetes Federation (IDF). Web site, 2020.

13. Omidian M, Djalali $M$, Javanbakht $H$. 
et al. Effects of vitamin D supplementation on advanced glycation end products signaling pathway in T2DM patients: a randomized, placebo-controlled, double blind clinical trial. Diabetol Metab Syndr, 2019, 11(1), 86

14. Abdallah A, Moustafa N, Soliman S. et al. Relationship of vitamin $D$ level and microalbuminuria in type 2 diabetic patients. Scientific J of Al-Azhar Med Faculty, Girls, 2019, 3(1), 205-212.

15. Mogahed MM. Vitamin D status in patients with type-2 diabetes mellitus in Riyadh City, Saudi Arabia. Kasr Al Ainy Med J, 24(1), 19. Vitamin D status in patients with type-2 diabetes mellitus in Riyadh City, Saudi Arabia. Kasr Al Ainy Med J, 2018, 24:19-25.

16. Abdel Aaty T, Magallaa M, Abdel Moneim $\mathrm{H}$. et al. Serum Vitamin D Level in Type 2 Diabetic Subjects: Relation to Glycemic Control, Insulin Resistance and Proinflammatory Markers. J. high inst. pub health, 2017, 47(2), 62-68.

17. Ahmadi N, Mortazav M, Iraj B. et al. Whether vitamin $\mathrm{D} 3$ is effective in reducing proteinuria in type 2 diabetic patients? J Res. Med. Sci, 2013, 18(5), 374-377.

18. Stefikova K, Spustova V, Krivosikova Z. et al. Insulin resistance and vitamin $D$ deficiency in patients with chronic kidney disease stage 2-3. Physiol Res, 2011, 60(1), 149-155.

19. Batra HS, Sampath S, Kumar SA. et al. Study of Vit D Supplementation in Type 2 Diabetes Mellitus. Int J Contemporary Med Res. 2019, Volume 6 | Issue 8; 47. 